\title{
Les éléments traces métalliques dans la lagune Ebrié : distribution saisonnière, niveau de contamination et qualité environnementale des sédiments
}

\author{
Ama Valérie WOGNIN *, Yao Mathieu N'GUESSAN, Fori Jean Paul ASSALE, Ané \\ Maurice AKA, Aoua Sougo COULIBALY, Sylvain MONDE et Kouamé AKA \\ Université Félix Houphouët Boigny d'Abidjan, UFR-STRM, Département de géosciences marines, 22 B.P. 582 \\ Abidjan 22, Côte d'Ivoire. \\ *Auteur correspondant,E-mail : ama_valerie@yahoo.fr
}

\section{RESUME}

Cette étude porte sur l'évaluation de la contamination en PHE des sédiments de la lagune Ebrié. Des prélèvements saisonniers de sédiments superficiels ont été effectués dans les baies estuariennes. La fraction fine $(<63 \mu \mathrm{m})$ a été considérée pour l'extraction et le dosage des éléments traces métalliques $(\mathrm{Ni}, \mathrm{Cu}, \mathrm{Cd}$, et Zn). Ces sédiments présentent des teneurs largement supérieures à celles de la croûte continentale (UCC Upper Continental Crust). Les facteurs de contamination (FC) calculées indiquent que les sédiments étudiés présentent des niveaux de contamination élevés. La réponse à la variation saisonnière a été également établit. D'une manière générale, on enregistre les plus faibles teneurs en étiage et les valeurs maximales pendant la période des précipitations. La toxicité potentielle des sédiments due à ces PHE a été évaluée grâce aux valeurs guides sédimentaires ERL (Effect Range low) et ERM (Effect Range-Medium). Il ressort de cette évaluation que les sédiments de ces baies présentent un risque de toxicité important pour les organismes vivants dans ce système aquatique. Ce risque augmente lors de la remise en suspension des sédiments par les phénomènes naturels et/ou par les activités anthropiques avec les opérations de dragage par exemple.

(C) 2017 International Formulae Group. All rights reserved.

Mots clés : Eléments traces métalliques (ETM), variation saisonnière, ERL, ERM, lagune Ebrié.

\section{The potential harmful elements (PHE) in lagoon Ebrié: seasonal distribution, contamination level, and environmental quality of sediment}

\section{ABSTRACT}

This study concerns the evaluation of the contamination in PHE of the sediments of the Ebrié lagoon. Seasonal sampling of surface sediments was carried out in estuarine bays. The fraction of silts and clays $(<63$ $\mu \mathrm{m})$ was considered for the determination of trace metallic elements $(\mathrm{Ni}, \mathrm{Cu}, \mathrm{Cd}$, and $\mathrm{Zn})$. The ETM concentrations of these sediments are much higher than those of the continental crust (UCC Upper Continental Crust). The contamination factor (CF) indicates that the sediments studied have high levels of contamination. Seasonal variation response was also established. Generally, the lowest levels of low water and maximum values are recorded during the precipitation period. The potential toxicity of sediments due to these PHE was evaluated using the ERL (Effect Range Low) and ERM (Effect Range-Medium) sediment guide values. This 
assessment indicates that the sediments of these bays have a significant risk of toxicity to living organisms in this aquatic system. This risk increases when the sediments are resuspended by natural phenomena and / or by anthropogenic activities with dredging operations, for example.

(C) 2017 International Formulae Group. All rights reserved.

Keywords: Potential Harmful Elements (PHE), seasonal variation, ERL, ERM, lagoon Ebrié.

\section{INTRODUCTION}

Depuis quelques années, une dégradation de plus en plus importante de la qualité de nos écosystèmes aquatiques a été constatée (Jadal et al, 2002). Celle-ci semble être engendrée d'une façon ou d'une autre par la croissance démographique. Les rejets agricoles, urbains et industriels peuvent conduire à des niveaux de pollution, variables dans les écosystèmes marins côtiers (Smolders et al., 2003 ; Rao et al., 2007 ; D’Adamo et al, 2008).

Dans le milieu aquatique, une grande partie des composés d'origine anthropique ou naturelle tendent à s'accumuler dans les sédiments et, souvent, à se concentrer dans les réseaux trophiques aquatiques. Ils sont introduits dans les cours d'eau sous forme particulaire, dissoute et colloïdale par les activités industrielles, urbaines, agricoles, ou par les dépôts atmosphériques (Nguéssan, 2008). Les Potential Harmful Elements (PHE) qui ne se retrouvent qu'en concentrations traces dans l'eau, constituent des exemples de substances toxiques et hydrophobes, qui ont tendance à s'accumuler dans la phase sédimentaire.

En effet, contrairement aux autres polluants (polluants organiques), les éléments traces métalliques ou Potential Harmful Elements (PHE) ne sont pas biodégradables dans l'environnement. Une des conséquences la plus sérieuse de la persistance de ces polluants est leur accumulation dans les chaînes alimentaires (Nguéssan, 2008).

Il paraît donc nécessaire de prendre en compte le risque environnemental associé à la contamination des sédiments en caractérisant la quantité des PHE stockés dans ce compartiment ainsi que la toxicité globale qu'ils représentent, surtout pour une lagune dont les baies sont fortement anthropisées.

L'objectif de cette étude est d'établir un bilan de la qualité du milieu lagunaire Ebrié en utilisant une approche multi-saisonnière des PHE (Ni, Cu, $\mathrm{Zn}, \mathrm{Cd})$. La réalisation de cet objectif passe par l'évaluation saisonnière de la distribution, du niveau de contamination et de la toxicité potentielle des sédiments superficiels de la lagune.

\section{MATERIEL ET METHODES}

\section{Echantillonnage et traitement physique des sédiments}

De février 2014 à décembre 2014, trois (3) campagnes d'échantillonnage (étiage, précipitations et crue) ont été réalisées dans l'estuaire de la lagune Ebrié (Figure 1). Le prélèvement des sédiments s'est effectué au moyen d'une benne de type Van Veen.

$\mathrm{Au}$ laboratoire, les sédiments ont été séchés à l'étuve et délicatement homogénéisé dans un mortier en agate, en veillant à ne pas altérer les grains. L'ensemble issu du mortier est quarté puis tamisé à l'aide de tamis en nylon pour obtenir 3 fractions: la fraction < $63 \mu \mathrm{m}$; la fraction comprise entre $63 \mu \mathrm{m}$ et $2 \mathrm{~mm}$ et enfin la fraction $>2 \mathrm{~mm}$. La fraction des limons et argiles $(<63 \mu \mathrm{m})$ a été choisie pour les analyses car les particules fines sont des cibles privilégiées pour l'adsorption des éléments naturels et plus particulièrement pour les éléments traces en raison de leur grande surface spécifique et de leurs grandes forces d'attraction ionique. 


\section{Protocole d'extraction et de dosage}

Pour chaque échantillon, environ 100 $\mathrm{mg}$ de la fraction $<63 \mu \mathrm{m}$ a été prélevé pour la digestion, selon le protocole UNEP (2007). Le principe de cette méthode de digestion est basé sur la décomposition des sédiments par l'acide hydrofluorique (HF) en combinaison avec de l'eau régale $\left(\mathrm{HNO}_{3}: \mathrm{HCl} ; 1: 3, \mathrm{v} / \mathrm{v}\right)$ à chaud. Les métaux ont été dosés par Spectrométrie d'Absorption Atomique (SAA) électrothermique à l'aide d'un Varian (model AA-20). La qualité des dosages a été validée en réalisant des blancs d'extraction, qui ont subi les mêmes traitements que les échantillons, mais également en vérifiant la teneur métallique d'un sédiment certifié de référence CRM BCSS-1 fourni par la Division de standards de chimie analytique marine du Canada. Les étalons pour la courbe d'étalonnage ont été préparés avec les mêmes réactifs que les échantillons.

\section{Calcul du facteur de contamination}

Pour l'évaluation du niveau de contamination en PHE des sédiments étudiés, nous avons eu recours au calcul du facteur de contamination. Le calcul de ce facteur fait appel au fond géochimique. Nous avons considéré les valeurs références pour la lagune Ebrié (Coulibaly et al., 2010). Le facteur de contamination est calculé à partir de la formule suivante :

$\mathbf{F C}=\mathbf{C}_{\mathrm{PHE}} / \mathbf{C}_{\text {fond géochimique }}$

C PHE: Concentration du PHE dans le sédiment

$\mathrm{C}$ fond géochimique: Valeur PHE du fond géochimique
Une classification s'en suit, fonction de la variation des valeurs du FC :

$\mathrm{FC}<1$ : Faible contamination

$1 \leq \mathrm{FC}<3$ : Contamination modérée

$3 \leq \mathrm{FC}<6$ Contamination considérable

$\mathrm{FC} \geq 6$ Très forte contamination

\section{Utilisation des valeurs guides sédimentaires (SQGs)}

De nombreux outils ont été créés afin de déterminer si les contaminants métalliques ou organiques associés aux sédiments peuvent affecter de manière négative les organismes aquatiques. Des valeurs guides sédimentaires (SQG, Sediment Quality Guideline) ont ainsi été obtenues à partir des données de terrain (indicateurs chimiques, écologiques et toxicologiques). Long et al. (1995) ont défini des seuils de toxicité ERL (Effect Range low) et ERM (Effect Range Medium). Ces seuils ou critères de toxicité ont été obtenus de manière empirique, c'est-à-dire en compilant les résultats de nombreuses études réalisées soit au laboratoire, soit en milieu naturel et pour lesquelles, le niveau de contamination et de toxicité des sédiments étaient disponibles. A partir de cette banque de données, ces auteurs ont défini pour chaque contaminant les concentrations pour lesquelles des effets biologiques sont rarement (en dessous des ERL), occasionnellement (entre les ERL et ERM) ou fréquemment rencontrés (au-dessus des ERM). De cette façon, les seuils de toxicité développés prennent en compte les effets dus au mélange de contaminants ainsi que de nombreuses caractéristiques du sédiment. Le Tableau 1 présente les seuils de toxicité pour les métaux étudiés. 


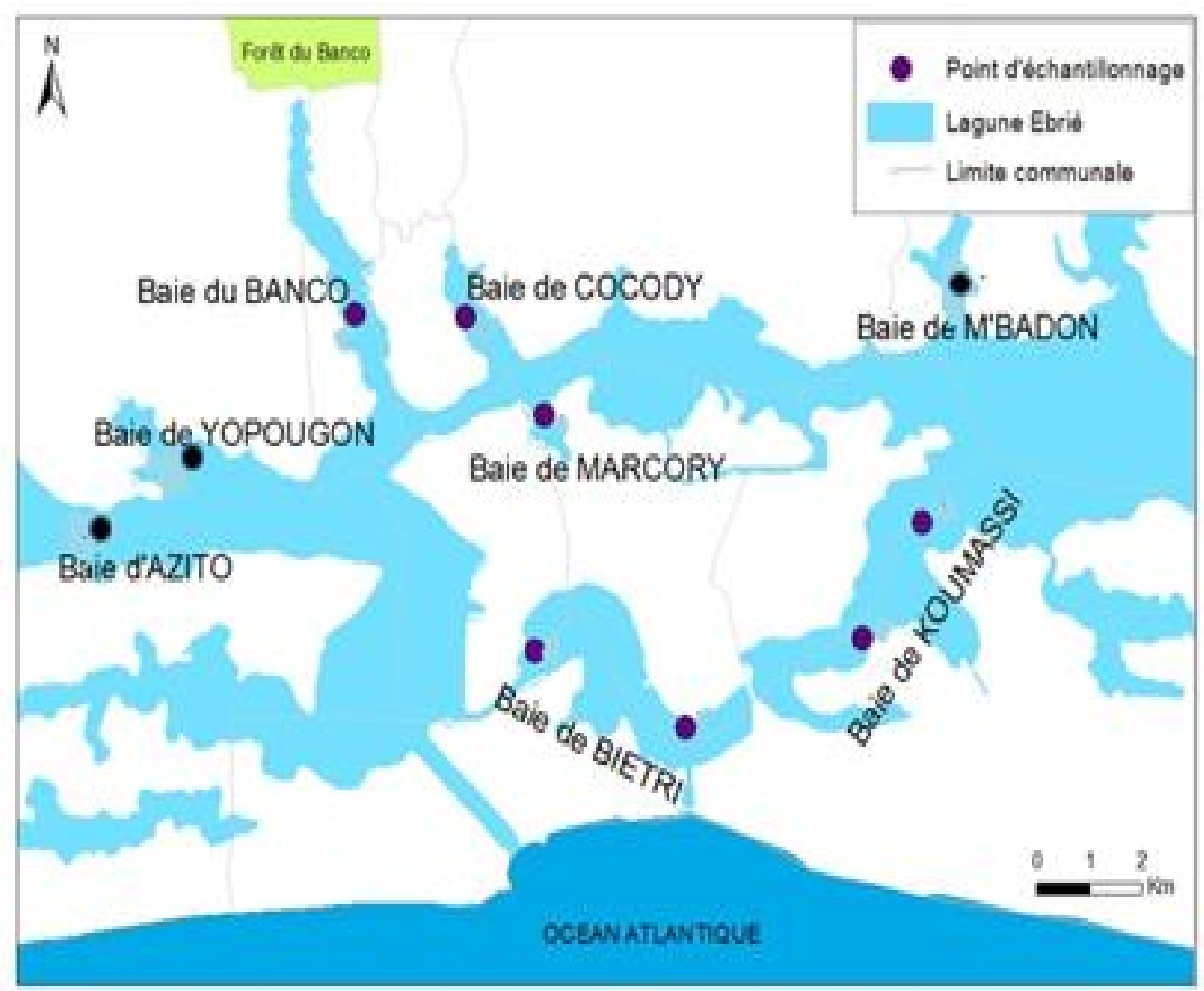

Figure 1 : Stations d'échantillonnage des sédiments de l'estuaire de la lagune Ebrié.

Tableau 1 : Seuils de toxicité ERL et ERM pour les $\mathrm{Cd}, \mathrm{Cu}, \mathrm{Ni}, \mathrm{Zn}$.

\begin{tabular}{lcc}
\hline Métal & ERL $(\mu \mathrm{g} / \mathrm{g})$ & ERM $(\mu \mathrm{g} / \mathrm{g})$ \\
\hline $\mathrm{Cd}$ & 1,2 & 9,6 \\
$\mathrm{Cu}$ & 34,0 & 270,0 \\
$\mathrm{Ni}$ & 20,9 & 51,6 \\
$\mathrm{Zn}$ & 150,0 & 410,0 \\
\hline
\end{tabular}




\section{RESULTATS}

Distribution saisonnière des PHE dans la lagune Ebrié

Les concentrations totales $\mathrm{du} \mathrm{Ni}, \mathrm{Cu}$, $\mathrm{Zn}$ et $\mathrm{Cd}$ des sédiments échantillonnés au cours des saisons de précipitations, d'étiage et de crue sont reportées dans le Tableau 2. Ces valeurs sont présentées avec à titre indicatif les valeurs de la croute continentale (UCC) pour chaque PHE. Il apparaît clairement que les concentrations de PHE dans la lagune Ebrié sont largement supérieures à ces dernières. Cela suggère une contribution anthropique.

La tendance qui se dessine, c'est l'augmentation des valeurs des PHE avec l'évolution des saisons, avec dans l'ordre croissant des teneurs: Etiage, crue et précipitations pour $\mathrm{Ni}, \mathrm{Cu}, \mathrm{Cd}(72,05,98,03$ et $110,01 \mu \mathrm{g} / \mathrm{g}$ pour le $\mathrm{Ni}$ dans la baie de Yopougon) (Figures 2, 3 et 4). Quant au Zn (Figure 5), cette tendance est moins nette. On enregistre, d'une station à l'autre des teneurs maximales ou minimales aussi bien en crue, en étiage qu'en période de précipitation. La variation spatiale des valeurs des PHE en fonction des saisons n'est pas homogène sur toute la lagune Ebrié. Cette hétérogénéité se perçoit aussi bien pour la totalité des PHE que pour un même élément (Figures 2, 3 et 4).

\section{Niveau de contamination}

Les résultats du calcul du FC pour les trois saisons (Tableau 3) montrent des valeurs supérieures à 1. Ce qui indique une contamination en PHE avérée, avec une augmentation des valeurs liée aux changements de saisons. Les valeurs de FC augmentent suivant cet ordre : étiage, crue, précipitations.

Les sédiments de la baie de Biétri présentent les plus fortes contaminations $(F C \geq 6)$ pour les PHE étudiés, particulièrement pour le $\mathrm{Cd}$. Les valeurs de FC obtenus en précipitations sont les plus fortes, avec des maximales de 16 pour le $\mathrm{Cd}$ dans la baie de Biétri. Suivent les valeurs de 14 et 12 toujours pour le Cd dans la baie de Biétri, en saison de crue. En étiage, les valeurs de FC varient de 8 à 10 pour la baie de Biétri.

La contamination sédimentaire dans la baie de M'Badon est modérée $(\mathrm{FC}=1)$, au cours de toutes les saisons, sauf pour le $\mathrm{Cu}$ $(\mathrm{FC}=2)$ pendant la période des précipitations. Pour les autres baies, la contamination en PHE est en générale considérable.

\section{Evaluation de la toxicité des PHE}

La toxicité potentielle des sédiments de la lagune Ebrié a été évaluée en comparant les PHE mesurés aux différentes valeurs guides sédimentaires (Figures 6, 7, 8, 9).

On observe pratiquement la même tendance dans l'évolution de la qualité des sédiments pour les 3 saisons (étiage, crue, précipitation), avec une augmentation des effets biologiques connexes à la contamination par les PHE étudiés $(\mathrm{Ni}, \mathrm{Cu}$, et $\mathrm{Cd}$ ), sauf pour le $\mathrm{Zn}$. Pour le $\mathrm{Zn}$ l'évolution saisonnière est variable, avec dans l'ordre croissant des effets biologiques: dans les baies d'Azito (précipitations, crue et étiage), de Cocody (crue, précipitations et étiage) et de Koumassi 1 (étiage, précipitations et crue) Koumassi 2 (crue, étiage et précipitations).

\section{Le nikel (Ni)}

Tous les échantillons étudiés montrent des valeurs supérieures au seuil minimal ERL et maximales ERM sauf les teneurs obtenus en étiage et en crue pour la baie de M'Badon. Les valeurs obtenues au cours de ces 2 saisons se situent entre ERL et ERM (Figure 6). Ce qui signifie que pendant ces périodes, les effets biologiques sont occasionnels. Pour la période des précipitations pour la baie de M'Badon et les autres baies étudiées, peu importe la saison, la contamination par le $\mathrm{Ni}$ entraîne des effets biologiques fréquents. 


\section{Le cuivre ( $\mathrm{Cu}$ )}

La quasi-totalité des teneurs mesurées se situe dans l'intervalle ERL - ERM, sauf la valeur crue obtenue pour la baie de M'Badon, qui est inférieure à ERL (Figure 7). Donc pour cette seule mesure, on n'observe aucun effet biologique néfaste, par contre pour tout le reste, les effets attendus sont occasionnels.

\section{Le cadmium (Cd)}

Les valeurs des concentrations mesurées se répartissent en 2 groupes en fonction des valeurs seuils de toxicité ERL et ERM (Figure 8). En dessous de ERL, Les sédiments de la baie de M'Badon au cours des 3 saisons ne constituent pas un danger, car toutes les mesures de $\mathrm{Cd}$ effectuées sont en dessous de ERL. Il en est de même pour les sédiments prélevés en étiage dans les baies du
Banco, de Marcory et de Koumassi 1, en crue pour la baie d'Azito.

Entre ERL et ERM, Pour les sites de Yopougon, Cocody, Biétry ( 1 et 2$)$ et Koumassi 2, les effets biologiques sont occasionnellement observés au cours de toutes les saisons.

\section{Le zinc (Zn)}

Les sédiments de la baie de M'Badon ne constituent pas un danger pour l'environnement, quant à la teneur en $\mathrm{Zn}$, peu importe la saison considérée. Pour les autres sites étudiés, la comparaison des teneurs mesurées pour les 3 saisons et des valeurs guides sédimentaires, montrent que les effets biologiques dus au $\mathrm{Zn}$ varient de façon occasionnelle à fréquent (Figure 9).

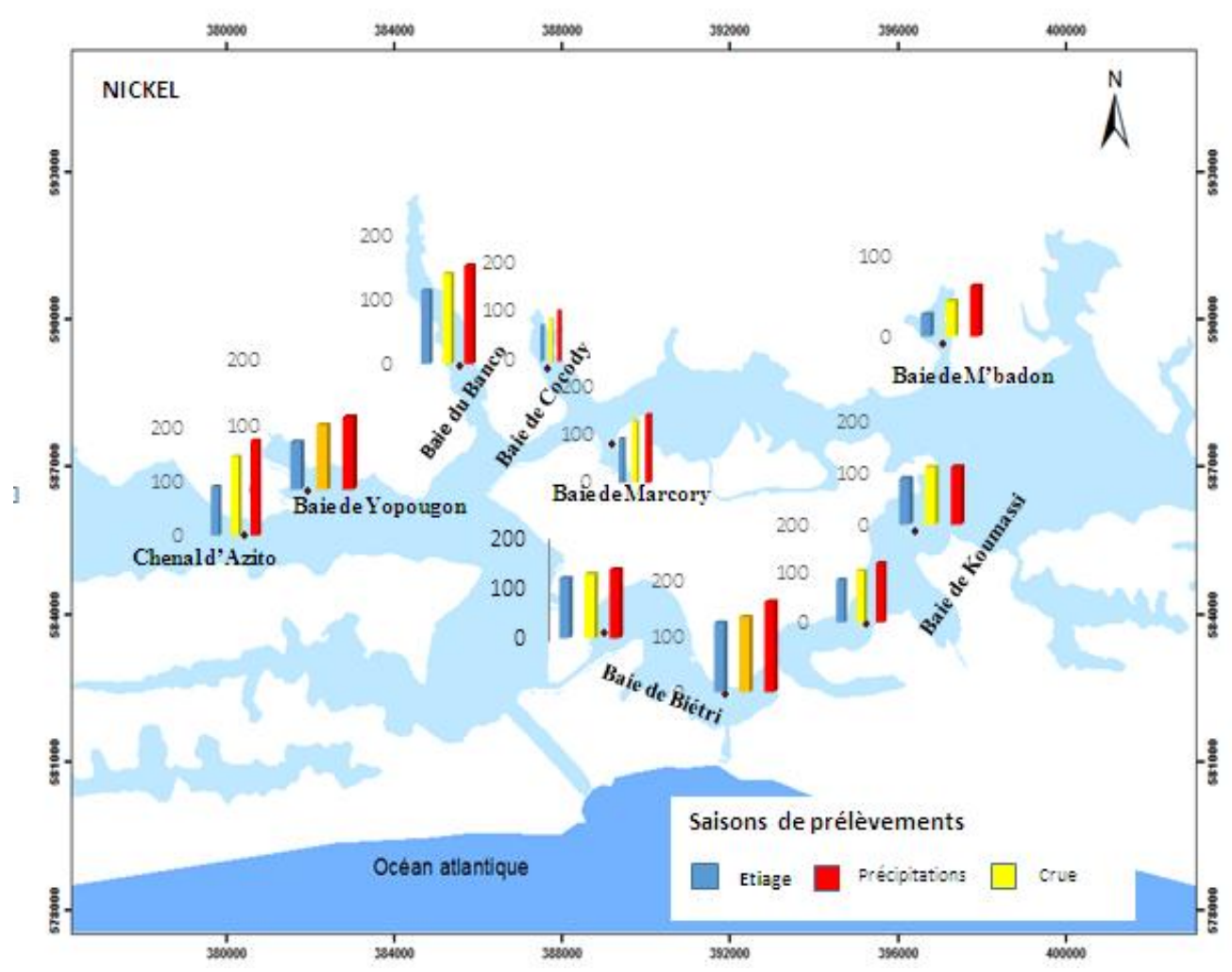

Figure 2 : Variation saisonnière du Ni dans les sédiments de l'estuaire de la lagune Ebrié. 
Tableau 2 : Concentration $(\mu \mathrm{g} / \mathrm{g})$ du Ni, Cu, Cd et $\mathrm{Zn}$ dans les baies de la lagune Ebrié.

\begin{tabular}{|c|c|c|c|c|}
\hline Stations & $\mathbf{N i}$ & $\mathrm{Cu}$ & Cd & $\mathbf{Z n}$ \\
\hline UCC & 19 & 14 & 0,1 & 52 \\
\hline \multicolumn{5}{|l|}{ Etiage } \\
\hline Azito & 118,97 & 115,05 & 1,43 & 545,32 \\
\hline Yopougon & 72,05 & 98,26 & 1,25 & 206,32 \\
\hline Banco & 113,51 & 154,97 & 0,86 & 380,79 \\
\hline Cocody & 72,08 & 117,06 & 1,71 & 406,85 \\
\hline Marcory & 90,09 & 110,52 & 0,94 & 231,57 \\
\hline Biétri 1 & 90,35 & 149,85 & 5,84 & 520,14 \\
\hline Biétri 2 & 132,14 & 127,21 & 4,6 & 689,39 \\
\hline Koumassi 1 & 85,14 & 73,27 & 0,85 & 218,32 \\
\hline Koumassi 2 & 88,11 & 58,25 & 2,89 & 236,49 \\
\hline M'Badon & 27,05 & 39,83 & 0,48 & 117,23 \\
\hline Moyenne & 88,949 & 104,427 & 2,085 & 355,242 \\
\hline \multicolumn{5}{|c|}{ Précipitations } \\
\hline Azito & 136,31 & 144,78 & 2,76 & 425,28 \\
\hline Yopougon & 110,01 & 113,03 & 2,32 & 404,72 \\
\hline Banco & 152,27 & 183,72 & 2,04 & 492,78 \\
\hline Cocody & 102,13 & 156,91 & 3,13 & 373,17 \\
\hline Marcory & 138,22 & 140,35 & 2,28 & 290,79 \\
\hline Biétri 1 & 174,37 & 157,37 & 9,27 & 703,83 \\
\hline Biétri 2 & 122,06 & 174,59 & 9,05 & 818,09 \\
\hline Koumassi 1 & 118,07 & 101,31 & 1,31 & 376,06 \\
\hline Koumassi 2 & 110,42 & 67,05 & 3,92 & 385,79 \\
\hline M'Badon & 61,24 & 53,21 & 0,78 & 150,22 \\
\hline Moyenne & 122,51 & 129,232 & 3,686 & 442,073 \\
\hline \multicolumn{5}{|l|}{ Crue } \\
\hline Azito & 127,54 & 132,09 & 0,85 & 505,71 \\
\hline Yopougon & 98,03 & 105,07 & 1,81 & 310,24 \\
\hline Banco & 139,39 & 162,37 & 1,29 & 453,38 \\
\hline Cocody & 84,17 & 122,36 & 2,96 & 245,07 \\
\hline Marcory & 125,37 & 128,24 & 2,03 & 261,04 \\
\hline Biétri 1 & 145,06 & 158,94 & 8,13 & 645,09 \\
\hline Biétri 2 & 159,07 & 158,14 & 6,9 & 769,75 \\
\hline Koumassi 1 & 103,19 & 86,38 & 1,98 & 421,27 \\
\hline Koumassi 2 & 109,36 & 75,94 & 3,42 & 207,23 \\
\hline M'Badon & 43,08 & 28,52 & 0,61 & 138,01 \\
\hline Moyenne & 113,426 & 115,805 & 2,998 & 395,679 \\
\hline
\end{tabular}




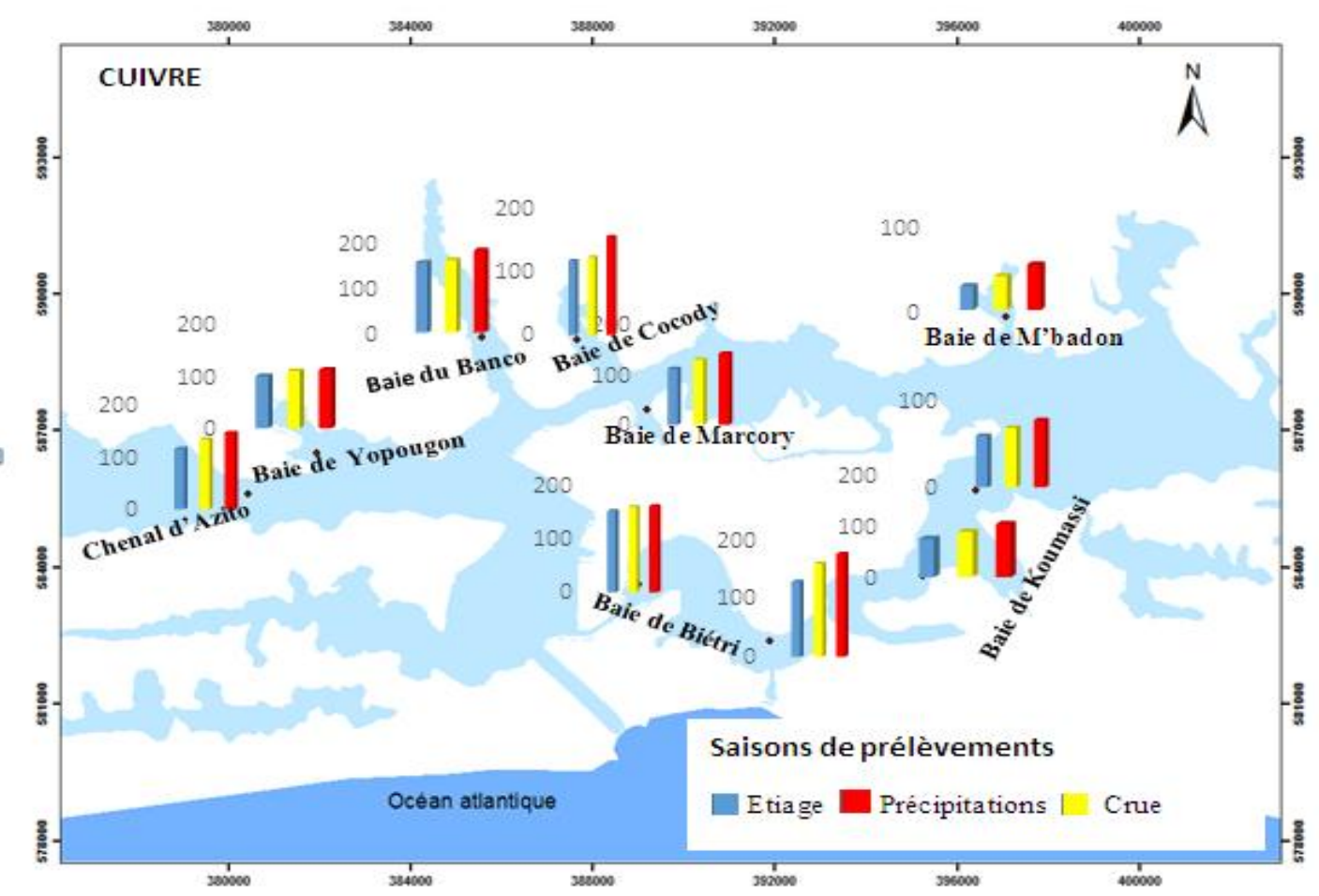

Figure 3 : Variation saisonnière du $\mathrm{Cu}$ dans les sédiments de l'estuaire de la lagune Ebrié.

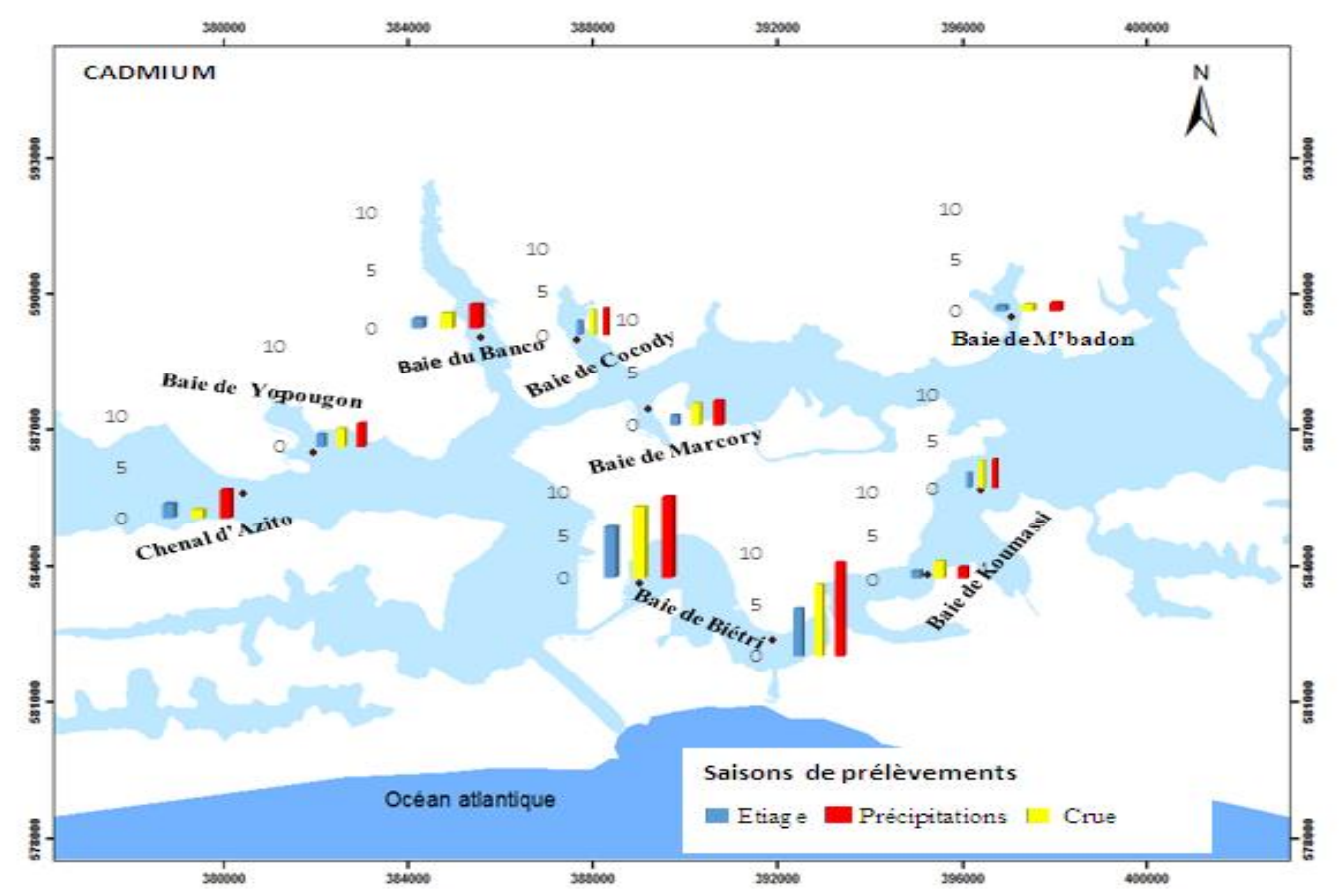

Figure 4 : Variation saisonnière du Cd dans les sédiments de l'estuaire de la lagune Ebrié 


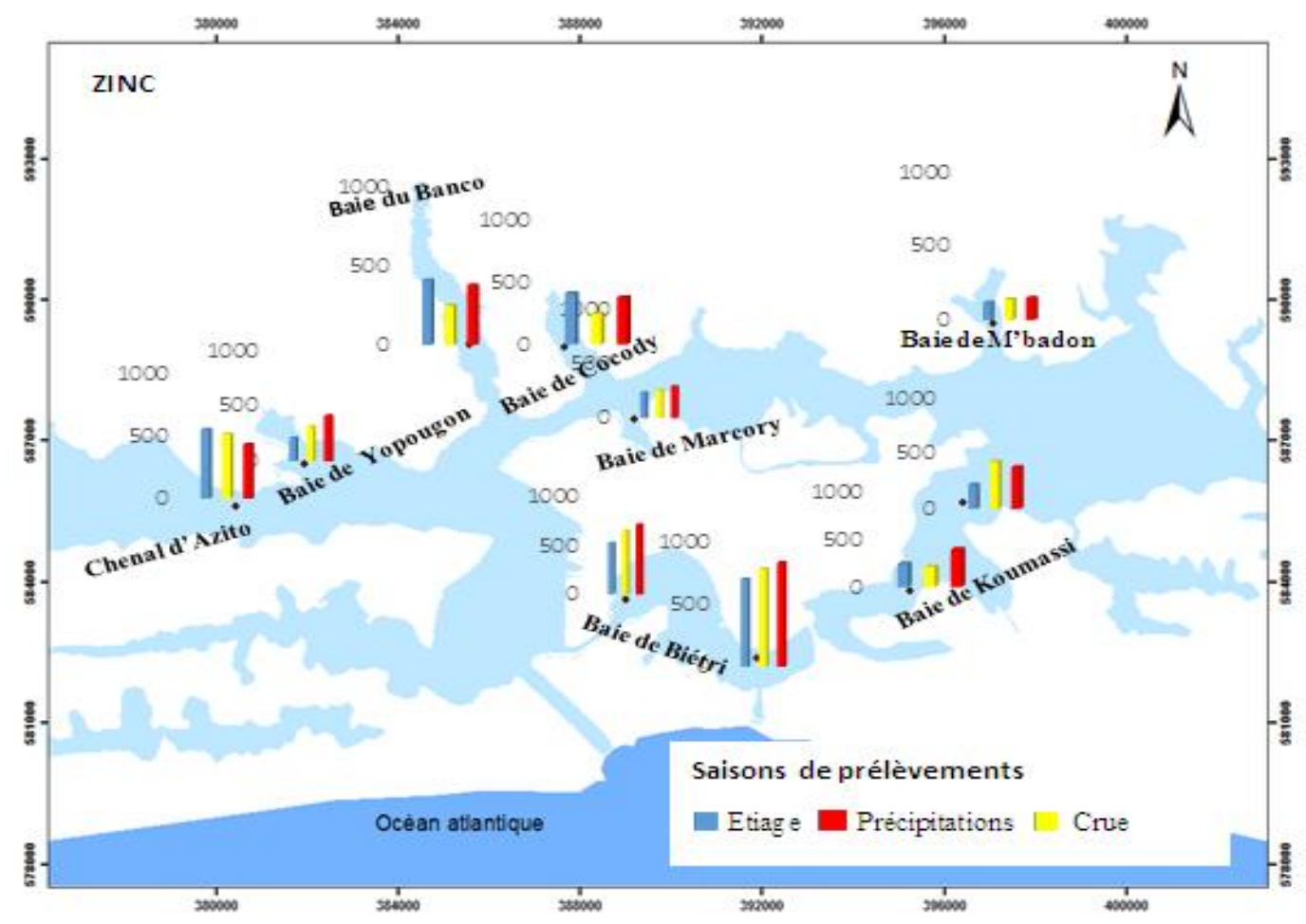

Figure 5 : Variation saisonnière du $\mathrm{Zn}$ dans les sédiments de l'estuaire de la lagune Ebrié.

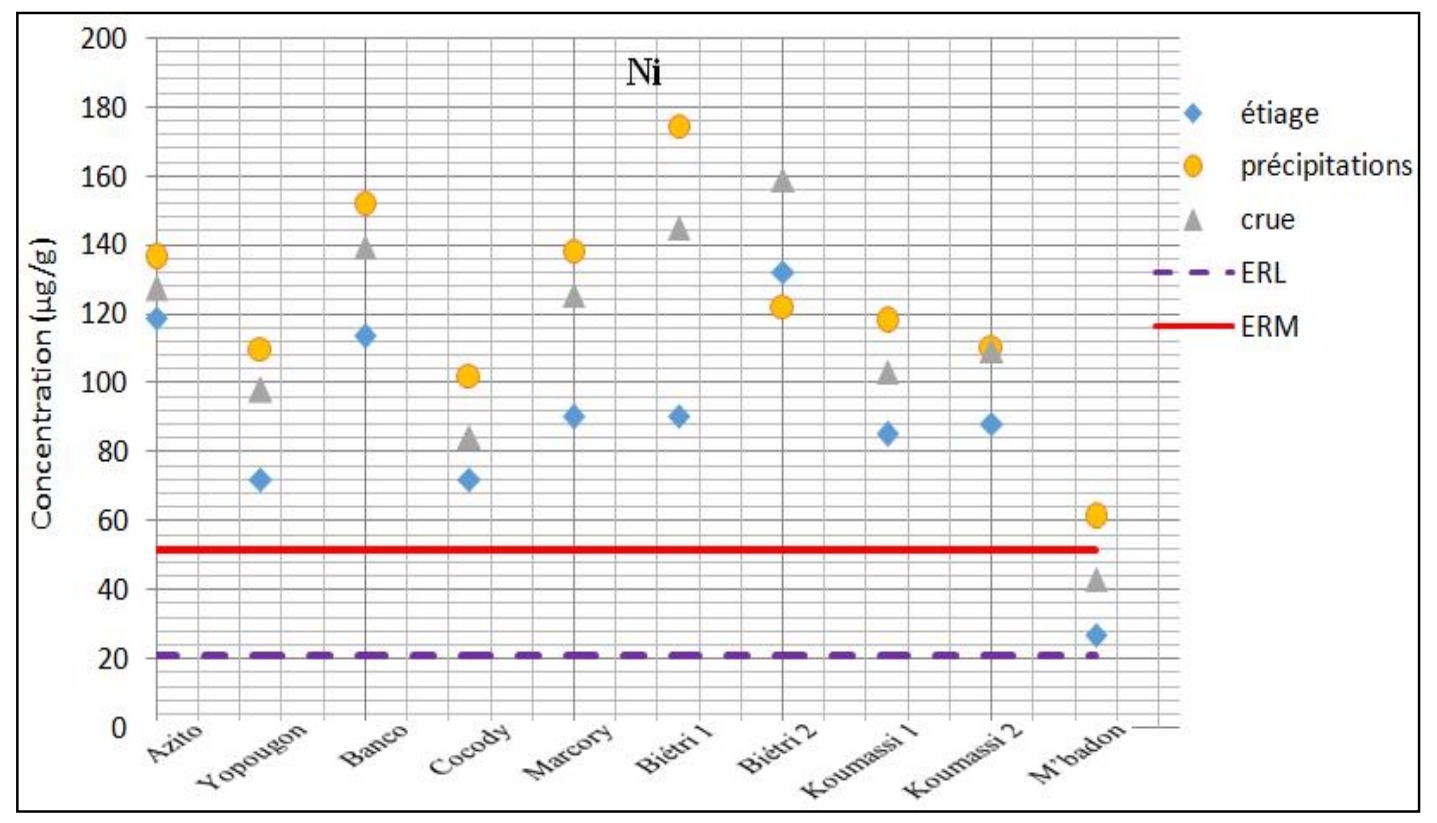

Figure 6 : Comparaison des valeurs guides d'effets biologiques (ERL et ERM) avec les teneurs de Ni dans les sédiments. 


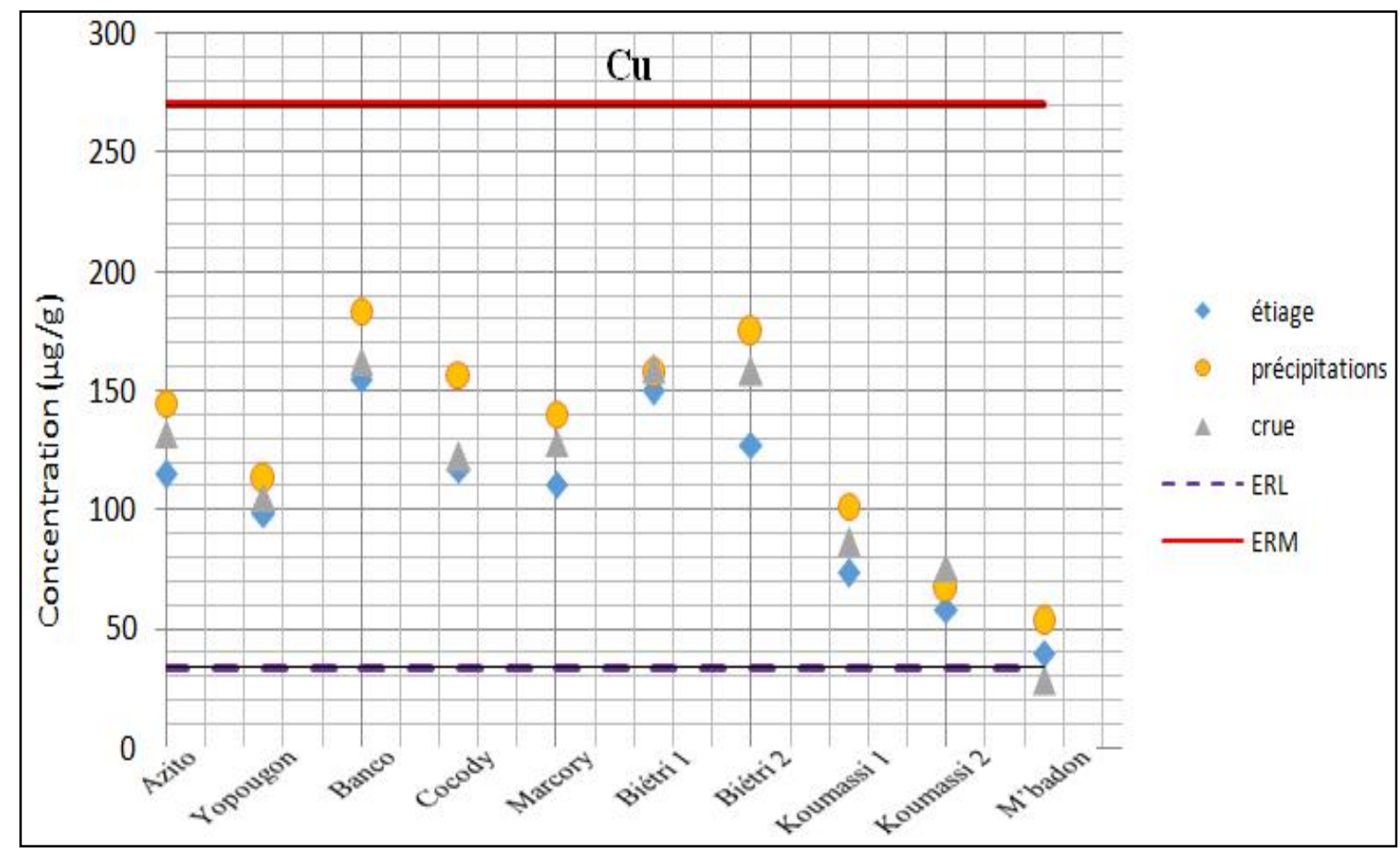

Figure 7 : Comparaison des valeurs guides d'effets biologiques (ERL et ERM) avec les teneurs de $\mathrm{Cu}$ dans les sédiments.

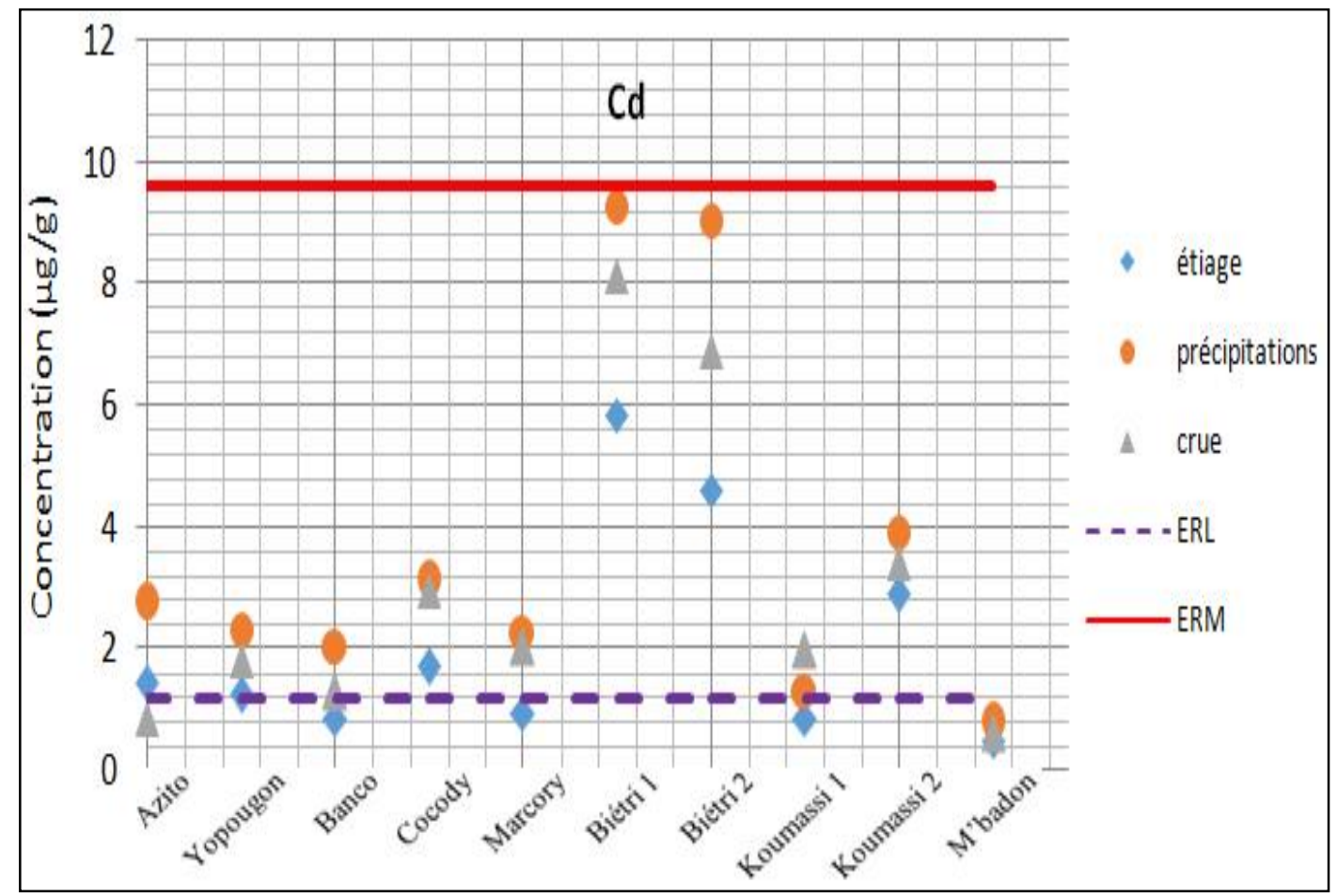

Figure 8 : Comparaison des valeurs guides d'effets biologiques (ERL et ERM) avec les teneurs de Cd dans les sédiments. 


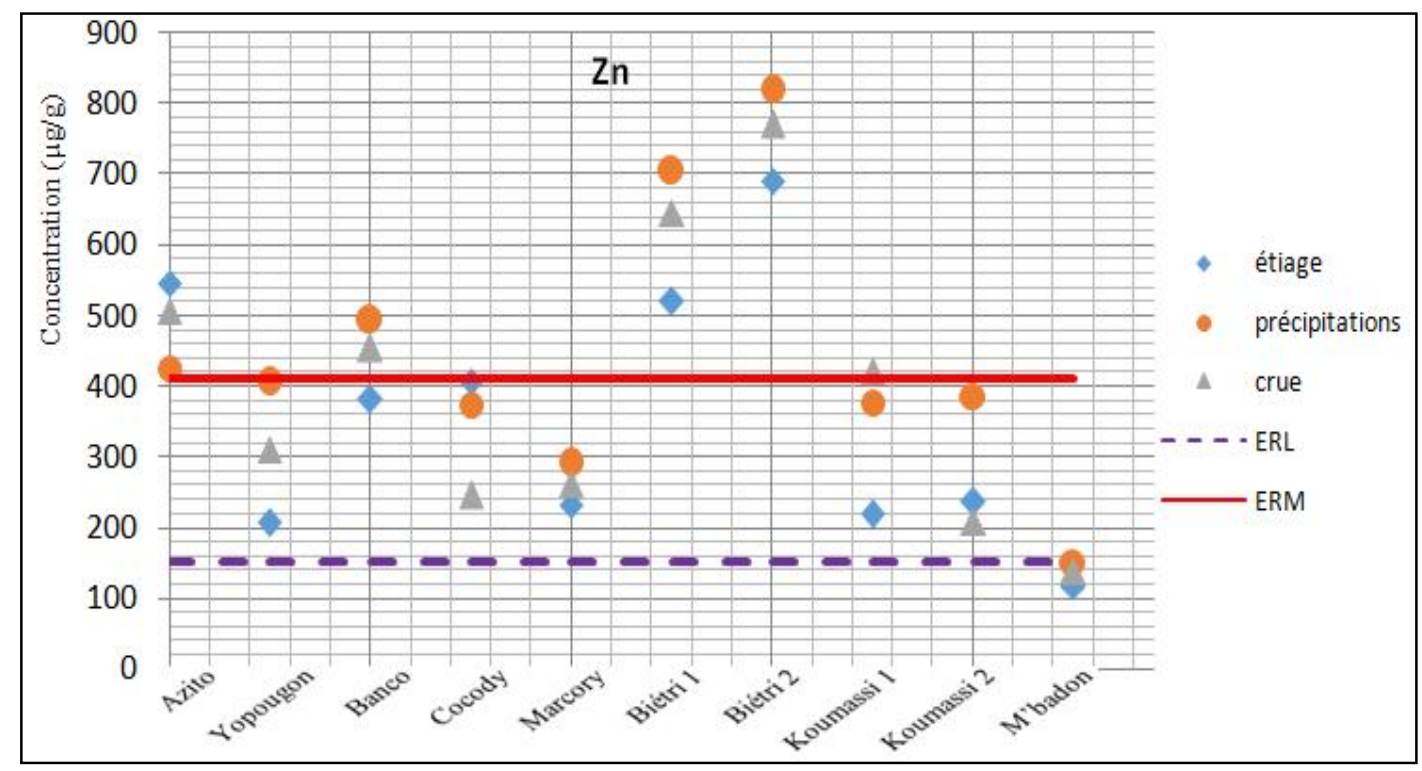

Figure 9 : Comparaison des valeurs guides d'effets biologiques (ERL et ERM) avec les teneurs de Zn dans les sédiments.

Tableau 3 : Facteurs de contamination (FC) des PHE considérés (A : fond géochimique).

\begin{tabular}{lllllllllllll}
\hline & \multicolumn{1}{c}{ Etiage } & \multicolumn{1}{c}{ Précipitation } & \multicolumn{1}{c}{ Crue } \\
\hline \multirow{3}{*}{ Azito } & $\mathrm{Ni}$ & $\mathrm{Cu}$ & $\mathrm{Cd}$ & $\mathrm{Zn}$ & $\mathrm{Ni}$ & $\mathrm{Cu}$ & $\mathrm{Cd}$ & $\mathrm{Zn}$ & $\mathrm{Ni}$ & $\mathrm{Cu}$ & $\mathrm{Cd}$ & $\mathrm{Zn}$ \\
Yopougon & 3 & 3 & 2 & 4 & 3 & 3 & 5 & 3 & 3 & 3 & 1 & 4 \\
Banco & 2 & 2 & 2 & 2 & 3 & 3 & 4 & 3 & 2 & 2 & 3 & 2 \\
Cocody & 3 & 4 & 1 & 3 & 4 & 4 & 4 & 4 & 4 & 4 & 2 & 3 \\
Marcory & 2 & 3 & 3 & 3 & 3 & 4 & 5 & 3 & 2 & 3 & 5 & 2 \\
Biétri 1 & 2 & 3 & 2 & 2 & 3 & 3 & 4 & 2 & 3 & 3 & 4 & 2 \\
Biétri 2 & 2 & 4 & 10 & 4 & 4 & 4 & 16 & 5 & 4 & 4 & 14 & 5 \\
Koumassi 1 & 3 & 3 & 8 & 5 & 3 & 4 & 16 & 6 & 4 & 4 & 12 & 6 \\
Koumassi 2 & 2 & 2 & 1 & 2 & 3 & 2 & 2 & 3 & 3 & 2 & 3 & 3 \\
M'Badon & 2 & 1 & 5 & 2 & 3 & 2 & 7 & 3 & 3 & 2 & 6 & 2 \\
\hline
\end{tabular}

\section{DISCUSSION}

Distribution saisonnière des teneurs des PHE dans les sédiments montre que le nickel dans les sédiments de la zone estuarienne de la lagune Ebrié pour la présente étude concorde avec ceux de Keumean et al. (2013) effectuée dans la lagune Ebrié au niveau du grau de Bassam (fleuve Comoé). Les teneurs observées sont tout aussi élevées. Cependant, cette zone n'est pas directement soumise aux actions des activités industrielles comme la zone estuarienne.

Les teneurs moyennes annuelles du cuivre et $\mathrm{du}$ cadmium obtenues dans la présente étude sont largement supérieures à celles énoncés par Affian et al. (2009), Soro et al. (2009), Coulibaly et al. (2010), et Kouassi (2014), dans la lagune Ebrié. Notons que nos échantillons ont été prélevés dans les baies directement soumises aux rejets industriels. 
Les travaux de Yao (2009) sur les sédiments des baies de la lagune Ebrié ont déterminé les valeurs maximales en zinc des sédiments pendant la saison d'étiage. Egalement Keumean et al. (2013) à l'issu d'analyses saisonnières sur les sédiments du grau de Bassam (Comoé), ont constaté que les teneurs de zinc des sédiments en période de crue sont beaucoup plus importantes qu'en période de fortes précipitations. A certaines stations, les valeurs maximales ont aussi été enregistrées en saison d'étiage. Cette grande variabilité spatiotemporelle dans la richesse des sédiments aquatiques en zinc est le fait de sa grande mobilité (Coulibaly et al., 2009). Le zinc migre plus facilement, donc un lessivage et un relargage efficace suffisent pour l'entraîner dans la colonne d'eau.

Sur le facteur de contamination, on note une intensification de la contamination en saison des précipitations et de crue, où les apports des eaux continentales sont importants. Les différents facteurs de contamination obtenus ont permis d'établir un ordre décroissant de contamination allant du métal le plus contaminant au moins contaminant: $\mathrm{Cd}>\mathrm{Cu}>\mathrm{Zn}>\mathrm{Ni}>\mathrm{Mn}$. Coulibaly et al. (2010) avaient pu établir aussi un gradient de contamination métallique allant de $\mathrm{Cd}$ à $\mathrm{Zn}: \mathrm{Cd}>\mathrm{Cu}>\mathrm{Ni}>\mathrm{Zn}$ sur les sédiments des baies d'Abidjan prélevés en janvier 2006 (saison d'étiage). De ces études, il ressort que le cadmium demeure le métal qui contamine plus les sédiments de la zone estuarienne de la lagune Ebrié.

La qualité environnementale des sédiments est ainsi affectée à toutes les saisons avec une prédominance pendant la saison des précipitations. En effet, pendant cette saison, le flux des eaux usées domestiques et industrielles, principales voies de transport de polluants dans la lagune, est plus intense (Monde, 2004). Les concentrations des métaux dans les sédiments deviennent plus importantes.

\section{Conclusion}

D'une manière générale, les PHE augmentent suivant cet ordre: étiage crue Précipitation à l'exception du zinc. On note pour le zinc, une évolution différente sans ordre apparent pour les baies de Cocody, Koumassi1 et Koumassi 2. Cependant, pour la baie d'Azito, les PHE augmentent de la saison des précipitations à la saison d'étiage.

L'étude de la contamination par les PHE montre un enrichissement anormal des sédiments superficiels des baies étudiées. La baie de Biétri est la plus contaminée (FC $\mathrm{cd}^{\mathrm{C}}=16$ en saison de précipitation). Cependant, La baie de M'Badon la moins contaminée des sites étudiées présente un facteur de contamination modéré $(\mathrm{FC}=2)$. Les effets biologiques induits par ces contaminations en PHE caractérisés par l'utilisation des valeurs guides sédimentaires (ERL et ERM) sont occasionnels et pour la plupart fréquents.

\section{CONFLIT D'INTERETS}

Les auteurs déclarent qu'ils n'ont aucun conflit d'intérêts.

\section{CONTRIBUTIONS DES AUTEURS}

AVW est le principal investigateur de l'étude et a participé à toutes les phases du travail. FJPA et AMA ont contribué à la collecte des données sur le terrain et à la détermination des résultats au laboratoire; YMN, ASC, et SM ont participé à l'interprétation des résultats, la lecture et la correction du manuscrit; KA a assuré la supervision générale des travaux.

\section{REMERCIEMENTS}

Nous adressons nos sincères remerciements au Centre Ivoirien Antipollution (CIAPOL) pour sa facilitation dans le traitement de nos échantillons.

\section{RÉFÉRENCES}

Affian K, Robin M, Maanan M, Digbehi

B, Djagoua E, Kouame F. 2009.

Heavy metal and polycyclic aromatic hydrocarbons in Ebrié lagoon sediments, Côte d'Ivoire.

Environmental Monitoring and Assessment, 159(1-4): 531-541. DOI: 10.1007/s10661-008-0649-z

Coulibaly AS, Monde S, Wognin AV, Aka K 2009. Analyse des Eléments Traces Métalliques (ETM) dans les baies estuariennes d'Abidjan en Côte d'Ivoire. Afrique Science, $\mathbf{0 5 ( 3 ) : \quad 7 7 - 9 6 .}$ http://www.afriquescience.info/document. php?id=1661 
Coulibaly AS, Monde S, Aka K. 2010. Biodisponibilité et Spéciations chimique des éléments traces métalliques dans un environnement lagunaire confine; la baie de Biétry. International Journal of Africain Studies, 4: 1-52. . DOI http://dx.doi.org/10.4314/ijbcs.v5i6.33

D’Adamo R, Di Stasio M, Fabbrochini A. 2008. Migratory crustaceans as biomonitors of metal pollution in their nursery areas. The Lesina lagoon (SE Italy) as a case study. Environ. Monit. Assess, 143: 15-24. DOI: 10.1007/s10661007-9944-3

Jadal M, El Yachioui M, Bennasser L, Fekhaoui M, Foutlane A. 2002. Qualité des eaux de l'estuaire de l'oued Oum Erbia (Maroc) et influence de la dynamique marégraphique. Eau l'Ind. Nuis, 256: 59-66.

Keumean NK, Bamba BS, Soro GN, Soro BS, Biémi J. 2013. Concentration en métaux lourds des sédiments de l'estuaire du fleuve Comoé à Grand-Bassam (Sud-Est de la Côte d'Ivoire). Journal of Applied Biosciences, 61: 4530-4539.

Kouassi NLB. 2014. Contribution à l'étude de la distribution et de la mobilité et le potentiel de toxicité des métaux comme le cuivre, le zinc et le cadmium dans les sédiments d'une lagune Ebrié estuaire tropical (Côte d'Ivoire). Thèse de doctorat, 158

Long ER, MacDonald DD, Smith SL, Calder FD. 1995. Incidence of adverse biological effects within ranges of chemical concentrations in marine and estuarine sediments. Environmental Management, 19: 81-97. DOI: 10.1007/BF02472006

Mondé S. 2004. Etude de la modélisation hydrodynamique de la circulation des eaux dans la lagune Ebrié (Côte d'Ivoire).
Thèse de Doctorat Université de Cocody (Abidjan), 364p

Nemati K, Bakar NKA, Sobhanzadeh MRE, Abas 2011. Speciation of heavy metals by modified BCR sequential extraction procedure in different depths of sediments from Sungai Buloh, Selangor, Malaysia. Journal of Hazardous Materials, 192(1) : 402-410. DOI: 10.1016/j.jhazmat.2011. 05.039

N'Guéssan YM. 2008. Dynamique des éléments traces dans les eaux de surface des bassins versants agricoles de Gascogne, thèse de Doctorat Université de Toulouse $253 \mathrm{p}$.

Rao JV, Kavitha P, Srikanth K, Usman PK et Rao TG. 2007. Environmental contamination using accumulation of metals in marine sponges, Sigmadocia fibulata inhabiting the coastal waters of Gulf of Mannar, India. Toxicol. Environ. Chem., 89: 487- 498. http://dx.doi.org/10.1080/0277224060115 0588

Soro G, Métongo B, Soro N, Ahoussi $\mathrm{K}$, Kouamé KF, Zade S, Soro T. 2009. Métaux lourds $(\mathrm{Cu}, \mathrm{Cr}$, Mn et $\mathrm{Zn}$ ) dans les sédiments de surface d'une lagune tropicale africaine : cas de la lagune Ebrié (Côte d'Ivoire). Int. J. Biol. Chem. Sci., 3(6): 1408-1427. http://www.ajol.info

Smolders R, Bervoets L, Wepener V, Blust R, 2003. A conceptual framework for using mussels as biomonitors in whole effluent toxicity. Hum. Ecol. Riskassess, 9: 741760. http://dx.doi.org/10.1080/713609965

YAO KM. 2009. Contribution à l'étude des paramètres physico-chimiques des eaux de la lagune Ebrié dans la zone d'Abidjan (Côte d'Ivoire). Thèse de Doctorat, Université de Cocody, 182p. 